\title{
PERCEPÇÃO E ENFRENTAMENTO DA DOR EM CRIANÇAS E ADOLESCENTES COM FIBROMIALGIA JUVENIL E ARTRITE IDIOPÁTICA JUVENIL POLIARTICULAR
}

Pain perception and pain coping mechanisms in children and adolescents with juvenile fibromyalgia and polyarticular juvenile idiopathic arthritis

\author{
Melissa Mariti Fraga ${ }^{a}$ (D), Maria Teresa Terreria (D), Rafael Teixeira Azevedo ${ }^{\circledR}(\mathbb{0}$, \\ Maria Odete Esteves Hilário ${ }^{a}$ (D), Claudio Arnaldo Len ${ }^{a, *}$ (1)
}

\section{RESUMO}

Objetivo: Mensurar e comparar a dor musculoesquelética em pacientes com fibromialgia juvenil (FMJ) e em pacientes com artrite idiopática juvenil poliarticular (AIJ); e avaliar e comparar a percepção e o enfrentamento da dor.

Métodos: Foram avaliados, em estudo transversal, 150 crianças e adolescentes (e seus respectivos pais), divididos em três grupos: FMJ, AlJ e controles saudáveis. A mensuração e o enfrentamento da dor foram realizados por meio de instrumentos específicos. Para a avaliação da percepção da dor, desenvolveram-se três vinhetas com simulação de situações que pudessem gerar dor: aplicação de injeção, queda de bicicleta e isolamento social. Os pais e os pacientes responderam individualmente quanto à percepção da dor em cada situação.

Resultados: As maiores notas de dor, os menores escores de enfrentamento da dor, as maiores notas para a percepção da dor nas vinhetas e os piores índices de qualidade de vida relacionada à saúde foram observados nos pacientes com FMJ, quando comparados aos pacientes com AlJ e aos controles. O mesmo padrão foi observado com os respectivos pais.

Conclusões: Pacientes com AlJ e FMJ se comportam diferentemente em relação à percepção da dor e ao desenvolvimento de técnicas para o enfrentamento da dor. A dor deve ser avaliada sob diferentes perspectivas para um planejamento mais individualizado e efetivo do tratamento desses pacientes.

Palavras-chave: Dor musculoesquelética; Artrite juvenil; Fibromialgia; Percepção da dor; Adaptação psicológica.

\section{ABSTRACT}

Objective: To measure and compare musculoskeletal pain in patients with juvenile fibromyalgia (JFM) and polyarticular juvenile idiopathic arthritis (JIA), and to evaluate and compare pain perception and pain coping mechanisms in these patients.

Methods: In this cross sectional study, we evaluated 150 children and adolescents, and their respective parents, from 3 different groups: JFM, polyarticular JIA, and healthy controls. Pain intensity and pain coping mechanisms were measured using specific questionnaires. Pain perception was evaluated according to three illustrations simulating situations that might cause pain: a shot, a bicycle fall, and social isolation. The patients' parents also filled out the questionnaires and provided a pain score that matched their child's perception of pain for each illustration.

Results: The highest pain scores, the lowest pain coping strategy scores, the highest pain perception scores for all three illustrations, and the worse health related to quality of life indicators were observed in the JFM group, when compared to the JIA and control groups. The same pattern was observed with their parents.

Conclusions: Patients with JIA and JFM behave differently in relation to pain perception and the development pain coping mechanisms. Pain should be evaluated from different perspectives for an individualized and efficient treatment of patients.

Keywords: Musculoskeletal pain; Juvenile arthritis; Fibromyalgia; Pain perception; Psychological adaptation. 


\section{INTRODUÇÃO}

A dor musculoesquelética é uma das queixas mais comuns na faixa etária pediátrica. Muitos pacientes apresentam dor musculoesquelética idiopática (DMEI), caracterizada pela ausência de causas aparentes, caráter intermitente e, por vezes, incapacitante, por um período mínimo de três meses. ${ }^{1}$

Algumas séries de casos mostram que de 25 a $40 \%$ das crianças e adolescentes com síndrome de dor musculoesquelética crônica preenchem critérios para fibromialgia juvenil (FMJ)., ${ }^{2,3}$ Além disso, a frequência de FMJ em escolares é de 1,2 a $6,2 \%,{ }^{4,5}$ sendo mais comum em meninas, o que indica uma prevalência significativa dessa doença. Observa-se também um impacto nas atividades diárias, o que resulta em uma diminuição da qualidade de vida relacionada à saúde (QVRS) dos pacientes e de suas famílias.

A artrite idiopática juvenil (AIJ) é uma doença inflamatória crônica, caraterizada por artrite com duração mínima de seis semanas em, pelo menos, uma articulação. A dor é um achado frequente na AIJ, especialmente em pacientes com acometimento poliarticular (cinco ou mais articulaçôes inflamadas). Estudos prévios demonstraram que $86 \%$ das crianças com AIJ apresentaram dor de intensidade leve a moderada $^{6}$ e que a presença de dor e a AIJ também têm impacto sobre a QVRS.7-9

A dor, independentemente da etiologia, pode ser percebida, tolerada e enfrentada de formas diferentes pelos pacientes. No entanto, esses aspectos são pouco estudados quando se analisa FMJ e AIJ. ${ }^{10-12}$ Nosso estudo pioneiro teve como objetivos mensurar a dor de pacientes com FMJ e AIJ, bem como quantificar sua percepção da dor; e avaliar a adaptação à dor e o enfrentamento dessa condição por esses pacientes.

\section{MÉTODO}

Trata-se de um estudo transversal, realizado em um ambulatório especializado de um hospital terciário. Foram incluídos, consecutivamente, 100 pacientes com idades entre 8 e 18 anos: 50 pacientes com FMJ e 50 pacientes com AIJ poliarticular. Os pacientes com FMJ preencheram os critérios classificatórios para fibromialgia, conforme estabelecido pelo Colégio Americano de Reumatologia em $1990 .{ }^{13}$ Os pacientes com AIJ poliarticular preencheram os critérios diagnósticos da Liga Internacional Contra o Reumatismo. ${ }^{7}$ Todos os pacientes apresentavam um tempo mínimo de seguimento de seis meses.

O grupo controle foi constituído por 50 crianças e adolescentes selecionados aleatoriamente, por ordem de entrada, em um clube de lazer. Esse grupo foi pareado por sexo e idade e não apresentava história de doença inflamatória ou dor musculoesquelética crônica prévia.

Foram colhidos dados sobre idade de início, intensidade e duração da dor, presença de experiências dolorosas prévias relacionadas ou não à doença (cirurgias, internaçóes hospitalares, coleta de exames/punçôes venosas, fraturas, imobilizaçôes, traumas) e uso de medicação analgésica e/ou anti-inflamatória. Para os pacientes com AIJ, foram avaliados a atividade de doença e o número de articulaçóes ativas no momento da entrevista. Utilizamos a escala analógica visual numérica $(V A S){ }^{14}$ para avaliar a intensidade da dor, numerada de 0 a 10 .

Os pacientes informaram a intensidade da dor no momento da entrevista e a média de dor da última semana. Os pais indicavam a nota de dor que acreditavam que seus filhos apresentavam no dia da entrevista.

Para a avaliação da percepção da dor, desenvolvemos três vinhetas relacionadas a situações associadas à dor física ou psicológica:

1. dor física relacionada a trauma (queda de bicicleta);

2. dor relacionada a um procedimento médico (aplicação de injeção); e

3. dor de caráter emocional, devido à privação social (exclusão de um grupo de crianças).

As vinhetas foram elaboradas por um desenhista depois de uma reuniáo de consenso dos pesquisadores (Figura 1). Os personagens das vinhetas eram do sexo feminino e do sexo masculino, e os pacientes e controles foram orientados a atribuir uma nota de 0 a 10 (linha com extensáo de $10 \mathrm{~cm}$ ) de acordo com a percepção individual da dor, após a leitura de cada vinheta. Os pais também atribuíram uma nota de 0 a 10 para cada vinheta, avaliando a percepçáo da dor que seu filho poderia apresentar em cada situação. A vinheta foi apresentada individualmente a cada participante, uma por vez e mantendo a mesma sequência.

Para avaliar o enfrentamento da dor, foi utilizado o questionário Waldron/Varni Pediatric Pain Coping Inventory (PPCI), traduzido para a língua portuguesa. ${ }^{15}$ Essa ferramenta engloba as seguintes dimensôes: instrução autocognitiva ("digo a mim mesmo para ser corajoso"; "imagino que posso fazer a dor ou o machucado sarar por mim mesmo"; "finjo não sentir dor ou não ter machucado"; "digo a mim mesmo que tudo ficará bem; sei que posso fazer algo para melhorar a dor ou machucado"; "sei que posso pedir alguma coisa para melhorar a dor ou machucado"; "finjo que a dor não é táo ruim quanto realmente ela é"), atitudes de resolução ("vou para cama"; "peço por remédios"; "esfrego o ponto dolorido"; "respiro bem fundo"; "fico sentado e quieto"; "me deito"; "peço para ir ao médico"; 
"peço para alguém explicar por que sinto dor"; "coloco gelo ou calor nos pontos doloridos"; "vou dormir para me sentir melhor"), distraçâo ("penso em viajar ou nas férias"; "assisto à televisão"; "brinco com algum jogo"; "como ou bebo algo"; "tento não pensar na dor ou no machucado"; "ignoro a dor ou o machucado"; "penso em coisas boas"; "brinco com meu bichinho de estimação"; "leio um livro ou pinto um livro de colorir"; "falo sobre o que eu fiz no dia de hoje"), busca por apoio social ("peço por beijos e abraços"; "peço para alguém entender o quanto dói"; "brinco com meus amigos"; "peço para minha mãe, meu pai ou um amigo ficar sentado comigo"; "conto para a minha mãe ou para o meu pai"; "peço para ficar sozinho"; "aperto algo ou a mão de alguém ou alguma coisa"; "peço para alguém me falar que a dor diminuirá e que me sentirei melhor") e exagero de emoçóes/desamparo ("choro ou grito"; "penso apenas que só vai piorar"; "peço para a dor ir embora"; "tento ser forte e não dizer nada"; "fico bravo com as outras pessoas"; "penso que náo posso fazer nada para a dor passar").

Os pacientes com AIJ foram avaliados quanto à atividade clínica da doença, segundo os critérios de Wallace et al. ${ }^{16}$

Pacientes, controles e seus respectivos pais assinaram os termos de assentimento e consentimento. O estudo foi aprovado pelo Comitê de Ética em Pesquisa da Universidade Federal de São Paulo (CEP-UNIFESP 0309/10).
Inicialmente, os dados foram analisados descritivamente. Para as variáveis categóricas, foram apresentadas frequências absolutas e relativas. Para as variáveis numéricas, foram apresentadas medidas-resumo (média, mínimo, máximo, quartis e desvio padrão). A comparação de médias de dois grupos foi realizada via teste $t$ de Student para amostras independentes. Para mais de dois grupos, as médias foram comparadas por análise de variância (ANOVA), seguida de comparações múltiplas (comparaçóes de Duncan, para grupos com variâncias iguais, e C de Dunnett, para grupos com variâncias diferentes, em caso de evidência de diferença entre médias) para localizar as diferenças, em caso de normalidade (verificado via teste de Kolmogorov-Smirnov) dos dados. No caso de fuga da normalidade, foi utilizado o teste não paramétrico de Kruskal-Wallis, seguido pelo teste de Mann-Whitney (em caso de evidência de diferença entre médias) com correção de Bonferroni. Para a comparação de médias de amostras pareadas, foi utilizado o teste $t$ de Student para amostras pareadas. Para verificar associaçōes entre variáveis categóricas, foram utilizados os testes do qui-quadrado ou exato de Fisher em caso de amostras pequenas. As associaçóes lineares entre variáveis numéricas (escalas) foram analisadas via correlaçáo de Spearman. Para os cinco aspectos do PPCI, foi avaliada, pelo alpha de Cronbach, a consistência interna entre os itens que compóem cada um desses aspectos. Para todos os testes estatísticos foi utilizado um nível de significância de 5\%.

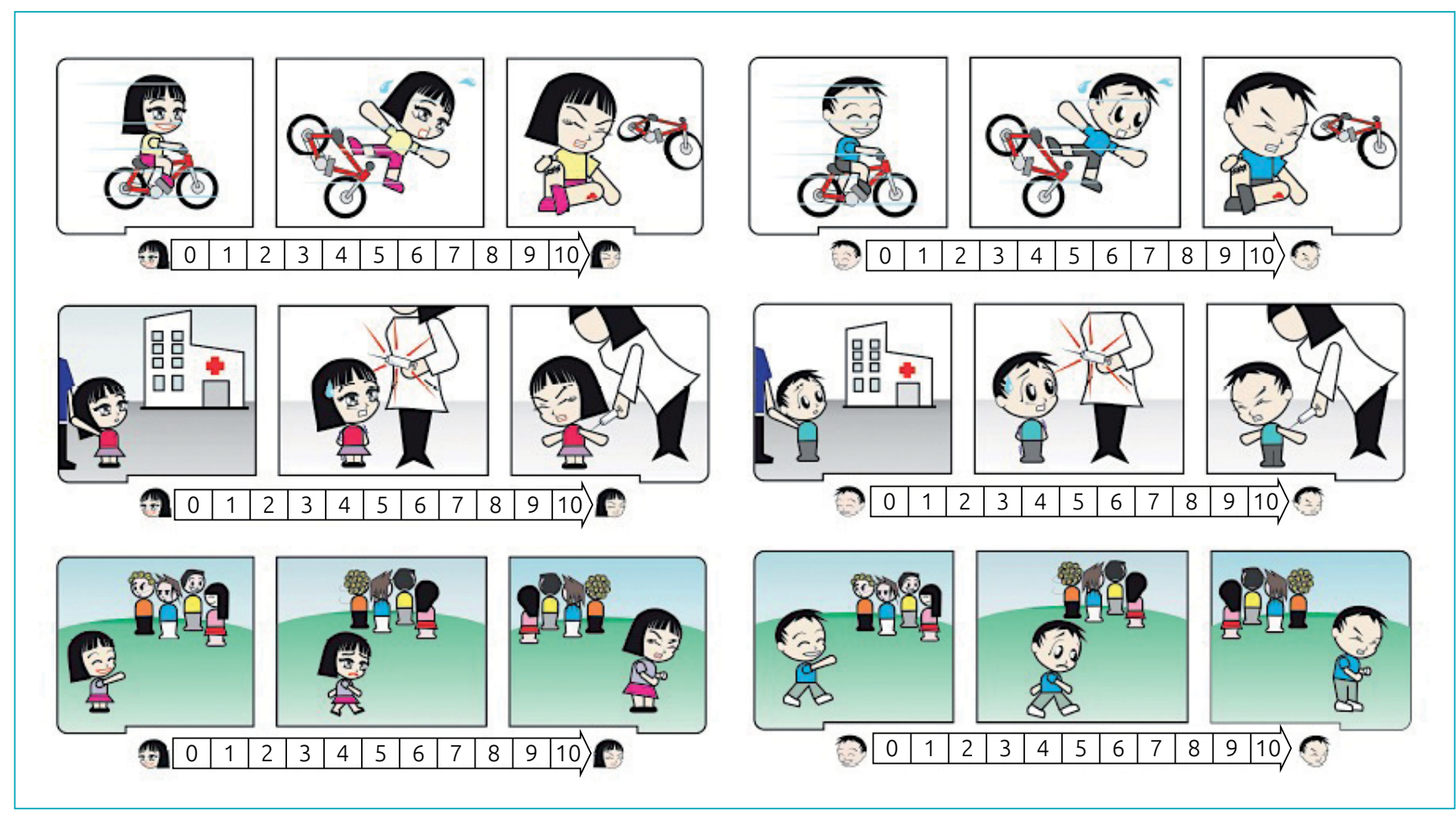

Figura 1 Vinhetas com situações de dor simulada: trauma após queda de bicicleta (primeira linha); aplicação de medicação parenteral: injeção (segunda linha) e privação social causada por um grupo de crianças (terceira linha). 


\section{RESULTADOS}

Foram realizadas 300 entrevistas com 150 pacientes e seus pais. Os dados clínicos e demográficos dos pacientes e os dados demográficos dos controles estão apresentados na Tabela 1. Observamos que os três grupos foram homogêneos quanto à classificação econômica, ao sexo e à idade do paciente no momento da entrevista.

Dos 50 pacientes com AIJ poliarticular avaliados (sete com fator reumatoide positivo), 29 (58\%) estavam com doença ativa no momento da avaliação.

\section{Notas de dor dos pacientes e controles}

Vinte e seis pacientes com FMJ (52\%) e 11 pacientes com AIJ poliarticular (22\%) apresentaram dor de forte intensidade (nota de dor $>8)$ na semana que antecedeu à entrevista ( $\mathrm{p}$-valor $<0,0001)$. Nove dos 50 pacientes do grupo FMJ (18\%) utilizaram analgésicos na semana que antecedeu à entrevista, enquanto 8 dos 50 pacientes com AIJ poliarticular (16\%) estavam em uso de medicamentos para a dor no momento da entrevista. Ainda com relação ao grupo de pacientes com AIJ, náo observamos diferença estatística entre a nota de dor no dia da entrevista e na semana antecedente à entrevista entre os pacientes que se encontravam com doença em atividade (presença de artrite) e em inatividade.

Houve diferença entre a média de idade de início de dor das crianças dos grupos FMJ e AIJ, sendo que os pacientes com AIJ apresentaram início de dor em idade mais jovem $(5,4 \pm 3,3$ versus $8,7 \pm 3,7 ; \mathrm{p}<0,0001)$ e maior duração da dor $(6,9 \pm 2,3$ versus 4,6 $\pm 1,9, \mathrm{p}<0,0001$ ) (Tabela 1).

Calculamos o índice de correlação intraclasse entre as notas de dor fornecidas pelas crianças e adolescentes do grupo FMJ e de seus pais. $\mathrm{O}$ valor encontrado foi de 0,716 , com intervalo de confiança de $95 \%$ (IC95\%) variando de 0,550 a 0,828 . Em relaçáo aos pacientes com AIJ, encontramos o valor de 0,658 , com IC95\% variando de 0,468 a 0,790 .

\section{Percepção da dor em pacientes e controles}

A Tabela 2 mostra os escores de percepção da dor em cada grupo entrevistado por meio das notas obtidas na aplicação das vinhetas sob o ponto de vista dos pacientes e de seus respectivos pais. O grupo de pacientes com FMJ apresentou as maiores médias e medianas de notas de dor nas três situaçóes simuladas apresentadas. Comparando-se as notas do grupo FMJ com as do grupo AIJ e as do grupo controle encontramos, respectivamente: 7,5 versus 4,7 versus 4,0 e $\mathrm{p}<0,0001$ para dor física; 8,5 versus 3,5 versus 7,1 e $\mathrm{p}<0,0001$ para dor relacionada a um procedimento médico; e 10,0 versus 6,0 versus 7,0 e $\mathrm{p}<0,0001$, para privação social. $\mathrm{Na}$ análise das vinhetas relacionadas ao procedimento médico e à privação social, foram encontradas notas distintas nos três grupos $(\mathrm{p}<0,0001)$, sendo que as maiores notas ocorreram no grupo de FMJ, seguido pelo grupo controle. Com relação à percepção da dor pelos pais, as notas também foram mais altas no grupo FMJ para as três situaçôes simuladas apresentadas.

Ao analisarmos separadamente cada situação de dor apresentada nas vinhetas, o grupo controle obteve notas mais altas do que os pacientes com $\mathrm{AIJ}$ na situação de dor por privaçáo social $(7,7$ versus 6,$0 ; \mathrm{p}<0,001)$ e dor relacionada a procedimento médico $(7,1$ versus 3,$5 ; \mathrm{p}<0,001)$.

O coeficiente da correlação de Spearman entre os escores de dor atribuídos por pais e filhos, de acordo com as vinhetas, está apresentado na Tabela 3. Observamos uma correlação

Tabela 1 Dados demográficos e clínicos dos pacientes com fibromialgia juvenil e artrite idiopática juvenil. Dados demográficos dos controles saudáveis. Nota de dor dos pacientes e controles segundo a perspectiva dos pacientes e cuidadores.

\begin{tabular}{l|c|c|c|c} 
& $\begin{array}{c}\text { Fibromialgia } \\
(\mathbf{n = 5 0 )}\end{array}$ & $\begin{array}{c}\text { Artrite idiopática } \\
\text { juvenil } \\
(\mathbf{n = 5 0 )}\end{array}$ & $\begin{array}{c}\text { Controles } \\
\text { saudáveis } \\
(\mathbf{n = 5 0 )}\end{array}$ & p-valor \\
\hline Sexo (feminino/masculino) & $43 / 7$ & $41 / 9$ & $42 / 8$ & 0,86 \\
\hline Idade de início da dor (anos)* & $8,7 \pm 3,7$ & $5,4 \pm 3,3$ & - & $<0,0001$ \\
\hline Duração da dor (anos)* & $4,6 \pm 1,9$ & $6,9 \pm 2,3$ & - & $<0,0001$ \\
\hline Idade do paciente na entrevista (anos)*Q & $13,5 \pm 2,7$ & $12,4 \pm 3,5$ & $12,8 \pm 2,3$ & 0,13 \\
\hline $\begin{array}{l}\text { Nota da dor no momento da entrevista sob o } \\
\text { ponto de vista dos pacientes e controles (0 a 10)* }\end{array}$ & $5,9 \pm 2,6$ & $2,1 \pm 2,4$ & $0,1 \pm 0,3$ & $<0,0001$ \\
\hline $\begin{array}{l}\text { Nota da dor na última semana sob o ponto } \\
\text { de vista dos pacientes e controles (0 a 10)* }\end{array}$ & $7,8 \pm 1,8$ & $3,2 \pm 2,6$ & $0,2 \pm 0,5$ & $<0,0001$ \\
\hline $\begin{array}{l}\text { Nota da dor no dia da consulta sob a } \\
\text { perspectiva dos pais (0 a 10)* }\end{array}$ & $5,9 \pm 2,9$ & $3,0 \pm 2,8$ & 0 & $<0,0001$ \\
\hline
\end{tabular}

*Média土desvio padrão; QANOVA com correção de Brown-Forsythe $\left(F_{2 \prime 132}=2,04\right.$; $p$-valor=0,1337), teste de Kolmogorov-Smirnov ( $p$-valor=0,4272) 
Tabela 2 Percepção da dor: comparações de médias ou medianas da nota de dor referida por grupo (fibromialgia juvenil, artrite idiopática juvenil e controles saudáveis) após a apresentação das vinhetas de traumatismo físico (queda de bicicleta), procedimento médico (aplicação de injeção) e privação social (isolamento por um grupo de crianças).

\begin{tabular}{|c|c|c|c|c|c|c|c|}
\hline & & Média & $\mathrm{DP}$ & Mediana & Mínimo & Máximo & p-valor \\
\hline \multicolumn{8}{|c|}{ Pacientes e controles } \\
\hline \multirow{3}{*}{$\begin{array}{l}\text { Traumatismo } \\
\text { físico }\end{array}$} & FMJ & $7,5^{(1)}$ & 1,8 & - & - & - & \multirow{3}{*}{$<0,0001^{*}$} \\
\hline & AlJ & $4,7^{(2)}$ & 2,6 & - & - & - & \\
\hline & Controle & $4,0^{(2)}$ & 2,3 & - & - & - & \\
\hline \multirow{3}{*}{$\begin{array}{l}\text { Procedimento } \\
\text { médico }\end{array}$} & FMJ & $8,5^{(1)}$ & 2,1 & - & - & - & \multirow{3}{*}{$<0,0001^{*}$} \\
\hline & AlJ & $3,5^{(3)}$ & 3,2 & - & - & - & \\
\hline & Controle & $7,1^{(2)}$ & 2,2 & - & - & - & \\
\hline \multirow{3}{*}{$\begin{array}{l}\text { Privação } \\
\text { social }\end{array}$} & FMJ & - & - & $10,0^{(A)}$ & 4 & 10 & \multirow{3}{*}{$<0,0001^{* *}$} \\
\hline & AlJ & - & - & $6,0^{(\mathrm{B})}$ & 1 & 10 & \\
\hline & Controle & - & - & $7,0^{(B)}$ & 0 & 10 & \\
\hline \multicolumn{8}{|l|}{ Pais } \\
\hline \multirow{3}{*}{$\begin{array}{l}\text { Traumatismo } \\
\text { físico }\end{array}$} & FMJ & $6,8^{(1)}$ & 2,2 & - & - & - & \multirow{3}{*}{$<0,0001^{*}$} \\
\hline & AlJ & $5,4^{(2)}$ & 2,9 & - & - & - & \\
\hline & Controle & $3,7^{(3)}$ & 2,6 & - & - & - & \\
\hline \multirow{3}{*}{$\begin{array}{l}\text { Procedimento } \\
\text { médico }\end{array}$} & FMJ & - & 2,6 & - & - & - & \multirow{3}{*}{$<0,0001^{*}$} \\
\hline & AlJ & - & 3,3 & - & - & - & \\
\hline & Controle & - & 2,9 & - & - & - & \\
\hline \multirow{3}{*}{$\begin{array}{l}\text { Privação } \\
\text { social }\end{array}$} & FMJ & - & - & $10,0^{(A)}$ & 5 & 10 & \multirow{3}{*}{$<0,0001^{* *}$} \\
\hline & AlJ & - & - & $6,5^{(\mathrm{B})}$ & 0 & 10 & \\
\hline & Controle & - & - & $7,0^{(C)}$ & 0 & 10 & \\
\hline
\end{tabular}

DP: desvio padrão; FMJ: fibromialgia juvenil; AlJ: artrite idiopática juvenil; (1), (2) e (3) apresentam médias distintas segundo comparações múltiplas de C de Dunnett com significância global de 5\%; (A), (B) e (C) apresentam médias distintas a uma significância global de $5 \%$ via testes de Mann-Whitney com correção de Bonferroni; *nível descritivo do teste da ANOVA; **nível descritivo do teste de Kruskal-Wallis.

Tabela 3 Percepção da dor: valor do coeficiente de correlação de Spearman entre os valores de dor atribuídos por pais e filhos para cada uma das três vinhetas (traumatismo físico/queda de bicicleta, procedimento médico/ injeção e privação social) e entre as vinhetas dentro dos grupos das crianças e adolescentes com fibromialgia juvenil, artrite idiopática juvenil e controle.

\begin{tabular}{l|c|c|c}
$\begin{array}{l}\text { Correlação } \\
\text { entre notas } \\
\text { de dor de pais } \\
\text { versus filhos }\end{array}$ & Fibromialgia & $\begin{array}{c}\text { Artrite } \\
\text { idiopática } \\
\text { juvenil }\end{array}$ & Controle \\
$\begin{array}{l}\text { Traumatismo } \\
\text { Físico }\end{array}$ & 0,256 & $0,467^{*}$ & $0,672^{*}$ \\
\hline $\begin{array}{l}\text { Procedimento } \\
\text { médico }\end{array}$ & 0,244 & $0,543^{*}$ & $0,675^{*}$ \\
\hline Privação social & 0,200 & $0,468^{*}$ & $0,596^{*}$ \\
\hline
\end{tabular}

estatisticamente significante entre as notas de pacientes com AIJ e seus pais, o que não foi encontrado no grupo de FMJ, para o qual a correlação foi baixa, mostrando que os pais não têm a mesma percepção da dor quando comparados aos seus filhos.

\section{Enfrentamento da dor pelos pacientes}

A Tabela 4 mostra o resumo e a comparação de médias dos itens instrução autocognitiva, atitudes de resoluçáo, distração, busca por apoio social e exagero de emoçóes/desamparo do questionário PPCI por grupo de pacientes e controle. Observou-se que as médias nos itens instrução autocognitiva e distraçáo foram similares entre os grupos FMJ e AIJ (7,6 versus 6,4), e ambas se apresentaram superiores às do grupo controle $(3,8)$. Observa-se, ainda no questionário PPCI, que os pacientes do grupo AIJ apresentaram escores maiores nos itens: atitudes de resoluçáo, busca por apoio social e exagero de emoçôes/desamparo. O grupo controle apresentou as menores médias. 


\section{DISCUSSÃO}

No presente estudo inédito avaliamos e comparamos dois aspectos relevantes para os clínicos habituados ao acompanhamento de crianças e adolescentes com dor musculoesquelética: percepção e enfrentamento da dor.

A percepção da dor é o processo pelo qual o organismo interpreta e organiza as sensaçóes para atribuir um significado. A percepção da dor não está relacionada diretamente com a atividade de doença (presença ou não de artrite ou número de pontos dolorosos no corpo), e sim a um conjunto de fatores, como experiências prévias do indivíduo, contexto social, estado emocional, sexo, raça, idade e cultura. ${ }^{17}$

A avaliação da percepção dolorosa, realizada por meio da apresentação das vinhetas de situaçôes simuladas, mostrou que o grupo FMJ apresentou as maiores notas ou a maior percepção dolorosa para as três situaçóes apresentadas, quando comparado aos demais grupos. A percepção foi maior tanto para os pacientes como para seus pais. A correlação entre a percepção da dor de pais e filhos foi pobre nos três grupos, devendo ser considerado o ponto de vista dos pacientes.

$\mathrm{Na}$ comparação das três vinhetas dentro do mesmo grupo, podemos notar como as experiências prévias de situaçóes vividas podem influenciar na percepção dolorosa. Avaliando cada grupo isoladamente, observamos que, no grupo FMJ, as maiores notas para percepção da dor foram fornecidas para simulação de privação social, situação que esses pacientes conhecem devido às limitaçóes impostas pela síndrome dolorosa.

Com as vinhetas conseguimos observar dois aspectos:

1. as notas de percepção da dor no grupo FMJ são bem mais altas que nos demais grupos em todas as situaçóes simuladas;

2. a percepção da dor, analisando um mesmo grupo, é fortemente influenciada pela experiência pessoal de cada indivíduo.

Tabela 4 Medidas-resumo e comparação de médias dos itens: instrução autocognitiva, atitudes de resolução, distração, busca por apoio social exagero de emoções/desamparo, do questionário Pediatric Pain Coping Inventory por grupo de crianças e adolescentes com fibromialgia juvenil, artrite idiopática juvenil e controle.

\begin{tabular}{|c|c|c|c|c|c|c|} 
& Média & DP & Mínima & Máxima & Mediana & p-valo*
\end{tabular}

Instrução autocognitiva (0 a 14)

\begin{tabular}{l|l|l|l|l|l|l|}
\hline Fibromialgia juvenil & $7,6^{(1)}$ & 3,7 & 0,0 & 14,0 & 8,0 \\
\hline Artrite idiopática juvenil & $6,4^{(1)}$ & 2,7 & 1,0 & 13,0 & 7,0 \\
\hline Controle & $3,8^{(2)}$ & 4,2 & 0,0 & 14,0 & 2,0 & $<0,0001$
\end{tabular}

Atitudes de resolução (0 a 20)

\begin{tabular}{l|c|c|c|c|c|c|}
\hline Fibromialgia juvenil & $7,8^{(\mathrm{B})}$ & 3,9 & 0,0 & 15,0 & 8,5 \\
\hline Artrite idiopática juvenil & $11,1^{(\mathrm{A})}$ & 3,7 & 4,0 & 19,0 & 11,0 \\
\hline Controle & $5,8^{(\mathrm{C})}$ & 3,2 & 0,0 & 15,0 & 6,0 & $<000$ \\
\hline
\end{tabular}

Distração (0 a 18)

\begin{tabular}{l|l|l|l|l|l|l}
\hline Fibromialgia juvenil & $6,5^{(\mathrm{A})}$ & 4,1 & 0,0 & 18,0 & 6,5 \\
\hline Artrite idiopática juvenil & $7,6^{(\mathrm{A})}$ & 4,0 & 0,0 & 17,0 & 9,0 \\
\hline Controle & $3,4^{(\mathrm{B})}$ & 3,1 & 0,0 & 11,0 & 3,0 & $<0,0001$ \\
\hline
\end{tabular}

Busca por apoio social (0 a 18)

\begin{tabular}{l|l|l|l|l|l|l}
\hline Fibromialgia juvenil & $6,4^{(\mathrm{B})}$ & 3,8 & 0,0 & 18,0 & 6,0 \\
\hline Artrite idiopática juvenil & $8,8^{(\mathrm{A})}$ & 4,3 & 0,0 & 18,0 & 9,0 \\
\hline Controle & $4,4^{(\mathrm{C})}$ & 3,2 & 0,0 & 11,0 & 4,0 \\
\hline
\end{tabular}

Exagero de emoções/desamparo (0 a 12)

\begin{tabular}{l|l|l|l|c|c|c|}
\hline Fibromialgia juvenil & $5,0^{(2)}$ & 2,3 & 1,0 & 11,0 & 5,0 & 6,0 \\
\hline Artrite idiopática juvenil & $6,5^{(1)}$ & 2,5 & 1,0 & 12,0 & $<0,0001$ \\
\hline Controle & $3,2^{(3)}$ & 1,5 & 0,0 & 7,0 & 3,0 & \\
\hline
\end{tabular}

DP: desvio padrão; (A), (B) e (C) apresentam médias distintas segundo comparações múltiplas de Duncan com significância global de 5\%; (1), (2) e (3) apresentam médias distintas segundo comparações múltiplas de C de Dunnett com significância global de 5\%; *nível descritivo da ANOVA. 
No grupo AIJ, a maior nota obtida por meio das vinhetas foi para a simulação de isolamento social, seguida de trauma físico e, entâo, de injeção. Todas as crianças e adolescentes que compuseram o grupo AIJ foram submetidas a injeçóes, tanto para uso de medicação injetável regularmente para controle da atividade de doença como para coleta de exames. O significado positivo que a medicação injetável pode ter para esse grupo provavelmente influenciou na nota menor de percepção da dor. Do mesmo modo, a situação de isolamento e restrição física que a AIJ é capaz de gerar em determinados momentos pode ter influenciado na nota maior da vinheta de privação social.

O grupo controle apresentou as maiores notas para a percepção da dor na simulação de injeção, seguida de privação social e, só depois, do trauma de bicicleta. Crianças e adolescentes saudáveis não são submetidos constantemente a experiências como aplicação de medicação injetável. Muitas dessas crianças só foram submetidas à vacinação de rotina como forma de medicação injetável. Destacamos que esse achado é inédito, uma vez que não encontramos artigos direcionados para a mensuração da percepção da dor em situaçôes hipotéticas. O uso das vinhetas elaboradas em nosso estudo ainda não está bem estabelecido na prática clínica diária. Acreditamos que, com a implantação desse instrumento na rotina do nosso ambulatório, poderemos obter informaçôes úteis no desenho do plano de tratamento individual.

O estudo de Varni et al..$^{15}$ avaliou, em pacientes pediátricos com doença reumática que apresentavam dor musculoesquelética, a relação entre experiências prévias de dor e a utilização de técnicas de enfrentamento adotadas por eles. Segundo esse autor, estratégias de enfrentamento são a chave na relaçáo entre percepção da dor e estado funcional da criança. Quanto maior o número de ferramentas ou mecanismos de enfrentamento da dor que um indivíduo possuir, menor será a percepção dolorosa e melhor será a chance de manter-se funcional. Diversos pesquisadores já sugeriram que, na dor musculoesquelética, as estratégias adotadas pela criança para enfrentar a dor têm um importante papel na determinaçāo da qualidade de vida da própria criança. ${ }^{18,19}$

A AIJ, apesar de ser uma doença inflamatória, pode levar a um comprometimento estrutural, muitas vezes de difícil remissão, das articulaçôes, dos tendôes e dos músculos. Illowite et al. ${ }^{20}$ observaram que a inflamação articular seria responsável por apenas $10 \%$ da variaçáo nos escores de dor e não encontraram relação significativa entre subtipo de doença ou número de articulaçôes acometidas com os escores de dor. Em estudo realizado por Hagglund et al. ${ }^{21}$ não se observou associação entre gravidade de doença e notas de dor em crianças com AIJ. Em nosso grupo de AIJ, não encontramos diferença estatística para nota de dor entre os subgrupos ativos e inativos da doença, motivo pelo qual pudemos considerá-lo como um grupo único e homogêneo. Em muitos casos, os pacientes com AIJ apresentam pouca ou nenhuma dor, ${ }^{6}$ especialmente quando o tratamento é realizado logo no início do diagnóstico e de maneira adequada, com base em protocolos atuais. ${ }^{22} \mathrm{~A}$ dor no grupo de pacientes com AIJ foi relatada como transitória, por alguns minutos ao acordar e para iniciar atividade física.

Sabe-se que a dor é um fator preponderante nos pacientes com dor musculoesquelética associada à amplificação, como é o caso da FMJ. Em nosso estudo, o grupo FMJ apresentou as maiores notas de dor, quando comparado aos demais grupos. Mais de $50 \%$ dos integrantes do grupo FMJ relataram dor intensa com nota de dor superior a 8 na semana antecedente à entrevista. Por outro lado, no grupo AIJ esse número foi inferior (22\%). Essa diferença na proporção de pacientes com dor intensa entre os grupos veio a confirmar nossa impressão da prática diária, em que pacientes com FMJ apresentam mais queixas de dor intensa e persistente interferindo nas atividades diárias, quando comparados aos pacientes com AIJ.

Nossos dados mostram que os pais de pacientes com FMJ conseguem aferir a dor referida pelos seus filhos melhor do que os pais dos pacientes com AIJ. Pouchot et al., ${ }^{23}$ em estudo avaliando a percepção de familiares e médicos de 7.700 pacientes com artrite reumatoide, observaram que familiares tendem a hipervalorizar a dor do paciente, enquanto os médicos que os acompanhavam tendem a subestimar a intensidade da dor.

Sawyer et al..$^{24}$ observaram que as ferramentas para enfrentamento mais utilizadas pelas crianças com AIJ são instrução autocognitiva e atitudes de resoluçáo. Em nosso estudo, os pacientes com AIJ e FMJ utilizam técnicas de instrução autocognitiva e distraçâo de maneira mais eficiente que o grupo controle. Ainda, o grupo AIJ utilizou técnicas como atitudes de resoluçáo, busca por apoio social e exagero de emoçôes/ desamparo como enfrentamento da dor.

Thastum et al. ${ }^{25}$ estudaram a relação entre estratégias de enfrentamento da dor e experiências de dor prévias em crianças com AIJ. Esses autores concluíram que, quanto maior a nota de dor, maior a chance dessa criança utilizar mecanismos como o exagero de emoçóes e desamparo para o enfrentamento. Em nosso estudo, esse dado náo pode ser confirmado. Os pacientes com AIJ utilizaram um maior número de técnicas de enfrentamento, talvez porque esse grupo tenha sido exposto a situaçóes dolorosas por um tempo mais prolongado ou por terem sido orientados sobre algumas técnicas de enfrentamento da dor durante seu tratamento.

Uma das limitaçóes de nosso estudo é o fato de avaliarmos um dado subjetivo e pessoal como a dor, para a qual o padrão ouro é o autorrelato. Durante todo o estudo estivemos atentos para evitar interferências externas. Todas as entrevistas foram realizadas pelo mesmo pesquisador (MMF), sendo mantidas a 
ordem de aplicação dos questionários e a apresentação das vinhetas para todos os participantes. Todo o estudo foi realizado no mesmo espaço físico (ambulatório de reumatologia pediátrica).

Pacientes com AIJ e FMJ se comportam diferentemente em relaçáo à percepção da dor e ao desenvolvimento de técnicas para o enfrentamento da dor. Em nosso estudo, pacientes com AIJ apresentavam maior tempo de exposição à dor, quando comparados a pacientes com FMJ, já que o quadro álgico se iniciou em idade mais precoce no grupo da AIJ. Esse grupo desenvolveu uma maior quantidade de mecanismos para o enfrentamento da dor. $\mathrm{O}$ maior tempo de exposição à dor poderia ter contribuído para o auxílio no desenvolvimento das habilidades de enfrentamento da dor.

A dor tem origem multifatorial e sofre influência de diversos aspectos em sua manutenção. Devemos avaliar o paciente com dor sob diversas perspectivas. Mostramos, em nosso estudo, que não basta apenas mensurarmos a intensidade da dor, precisamos avaliar, de maneira profunda, outros aspectos, como a percepçáo e o enfrentamento da dor. Uma vez identificado o perfil de cada paciente, caberá a todos os integrantes da equipe multidisciplinar elaborar um plano terapêutico individualizado e capaz de aliviar a dor em seus múltiplos aspectos, com destaque para o físico, o emocional, o social e o cognitivo.

\section{Financiamento}

O estudo não recebeu financiamento.

\section{Conflito de interesses}

Os autores declaram não haver conflito de interesses.

\section{REFERÊNCIAS}

1. Sherry DD, Malleson PN. The idiopathic musculoskeletetal pain syndromes in childhood. Rheum Dis North Am. 2002;28:669-85.

2. Malleson PN, al-Matar M, Petty RE. Idiopathic musculoskeletal pain syndrome in children. J Rheumatol. 1992;19:1786-9.

3. Yunus MB, Masi AT. Juvenile primary fibromyalgia syndrome: a clinical study of 33 patients and matched normal controls. Arthritis Rheum. 1985;28:138-45.

4. Zapata AL, Moraes AJ, Leone C, Doria-Filho U, Silva CA. Pain and musculoskeletal pain syndromes in adolescents. J Adolesc Health. 2006;38:769-71.

5. Buskila D, Neumann L, Hershmann E, Gedalia A, Press J, Sukenik S, et al. Assessment of nonarticular tenderness and prevalence of fibromyalgia in children. J Rheumatol. 1993;20:368-70.

6. Sherry DD, Bohnsack J, Salmonson K, Wallace CA, Mellins E. Painless juvenile rheumatoid arthritis. J Pediatr. 1990;116:921-3.

7. Petty RE, Southwood TR, Manners P, Baum J, Glass DN, Goldenberg J, et al. International League of Associations for Rheumatology classification of juvenile idiopathic arthritis: second revision, Edmonton, 2001. J Rheumatol. 2004;31:390-2

8. Petty RE, Southwood TR, Baum J, Bhettay E, Glass DN, Manners $P$, et al. Revision of the proposed classification criteria for juvenile idiopathic arthritis: Durban, 1997. J Rheumatol. 1998;25:1991-4.

9. Klatchoian DA, Len CA, Terreri MT, Silva M, Itamoto C, Ciconelli $\mathrm{RM}$, et al. Quality of life of children and adolescents from São Paulo: reability and validity of the Brazilian version of the Pediatric Quality of Life Inventory version 4.0 Generic Core Scales. J Pediatr (RioJ). 2008;84:308-15.

10. Granges G, Littlejohn GO. Pressure pain threshold in pain-free subjects, in patients with chronic regional pain syndromes, and in patients with fibromyalgia syndrome. Arthritis Rheum. 1993;36:642-6.

11. Carli G, Suman AL, Biasi G, Marcolongo R. Reactivity to superficial and deep stimuli in patients with chronic musculoskeletal pain. Pain. 2002;100:259-69.

12. Truckenbrodt H. Pain in juvenile chronic arthritis: consequences for the musculoskeletal system. Clin Exp Rheumatol. 1993;11:S59-63.

13. Wolfe F, Smythe HA, Yunus MB, Bennett RM, Bombardier C, Goldenberg DL, et al. The American College of Rheumatology 1990 Criteria for the Classification of Fibromyalgia. Report of the Multicenter Criteria Committee. Arthritis Rheum. 1990;33:160-72.

14. Cline ME, Herman J, Shaw ER, Morton RD. Standardization of the visual analogue scale. Nurs Res. 1992;41:378-80.

15. Varni JW, Waldron SA, Gragg RA, Rapoff MA, Bernstein $\mathrm{BH}$, Lindsley $\mathrm{CB}$, et al. Development of the Waldron/Varni pediatric pain coping inventory. Pain 1996;67:141-50.

16. Wallace CA, Ruperto N, Giannini E, Childhood Arthritis and Rheumatology Research Alliance, Pediatric Rheumatology International Trials Organization, Pediatric Rheumatology Collaborative Study Group. Preliminary criteria for clinical remission for select categories of juvenile idiopathic arthritis. J Rheumatol. 2004;31:2290-4.

17. Tracey I, Mantyh PW. The cerebral signature for pain perception and its modulation. Neuron. 2007;55:377-91.

18. Kashikar-Zuck S, Zafar M, Barnett KA, Aylward BS, Strotman $D$, Slater SK, et al. Quality of life and emotional functioning in youth with chronic migraine and juvenile fibromyalgia. Clin J Pain. 2013;29:1066-72.

19. Tupper SM, Rosenberg AM, Pahwa P, Stinson JN. Pain intensity variability and its relationship with quality of life in youths with juvenile idiopathic arthritis. Arthritis Care Res (Hoboken). 2013;65:563-70. 
20. Illowite NT, Walco GA, Pochaczevsky R. Assessment of pain in patients with juvenile rheumatoid arthritis: relation between pain intensity and degree of joint inflammation. Ann Rheum Dis. 1992;51:343-6.

21. Hagglund KJ, Schopp LM, Alberts KR, Cassidy JT, Frank RG. Predicting pain among children with juvenile rheumatoid arthritis. Arthritis Care Res. 1995;8:36-42.

22. Beukelman T, Patkar NM, Saag KG, Tolleson-Rinehart S, Cron RQ, DeWitt EM, et al. 2011 American College of Rheumatology recommendations for the treatment of juvenile idiopathic arthritis: initiation and safety monitoring of therapeutic agents for the treatment of arthritis and systemic features. Arthritis Care Res (Hoboken). 2011;63:465-82.
23. Pouchot J, Le Parc JM, Queffulec L, Sichère P, Flinois A; Association Française des Polyarthritiques. Perceptions in 7700 patients with rheumatoid arthritis compared to their families and physicians. Joint Bone Spine. 2007; 74:622-6.

24. Sawyer MG, Whitham JN, Roberton DM, Taplin JE, Varni JW, Baghurst PA. The relationship between health-related quality of life, pain and coping strategies in juvenile idiopathic arthritis. Rheumatology (Oxford). 2004;43:325-30.

25. Thastum M, Herlin T, Zachariae R. Relationship of pain-coping strategies and pain-specific beliefs to pain experience in children with juvenile idiopathic arthritis. Arthritis Rheum. 2005;52:178-84. 